\title{
Autosomal Recessive Hypohidrotic Ectodermal Dysplasia
}

National Cancer Institute

\section{Source}

National Cancer Institute. Autosomal Recessive Hypohidrotic Ectodermal Dysplasia. NCI

Thesaurus. Code C84580.

A rare autosomal recessive disorder characterized by developmental abnormalities of the skin, sweat glands, hair and nails. Patients have a reduced ability to sweat. Other signs and symptoms include hypotrichosis and teeth malformations. 